\title{
Cases of Opsi Syndrome Still Candidate for Medical ICU
}

\author{
Gulbin Aygencel $^{1}$, Murat Dizbay ${ }^{2}$, Melda Aybar Turkoglu ${ }^{3}$ and Ozlem Guzel Tunccan ${ }^{4}$ \\ ${ }^{1}$ Department of Internal Medicine, Division of Critical Care Medicine; Gazi University Faculty of Medicine; ${ }^{2}$ Department of Clinical \\ Microbiology and Infectious Disease, Gazi University Faculty of Medicine; ${ }^{3}$ Department of Internal Medicine, Division of Critical Care \\ Medicine,Gazi University Faculty of Medicine; ${ }^{4}$ Department of Clinical Microbiology and Infectious Disease, Gazi University Faculty of \\ Medicine; Ankara, Turkey
}

\begin{abstract}
Splenectomized patients are likely to suffer from severe infections, such as sepsis and meningitis, which is called overwhelming postsplenectomy infection (OPSI) syndrome. It seems to be more common in children, but occurs at all ages. The risk is greatest in the early months and years after operation, but never disappears entirely. The course is rapid, the clinical symptoms are serious, and the prognosis is very poor. In this paper, three cases of OPSI syndrome are described, in which infection developed 8, 8 and 15 years after splenectomy; two of the patients died. With the help of these case reports, we want to again emphasize the importance of vaccination, antibiotic prophylaxis and seeking earlier medical attention in splenectomized patients.
\end{abstract}

Key-Words: Overwhelming postsplenectomy infection (OPSI) syndrome, splenectomy.

The risk of severe and sometimes fatal infection after splenectomy is well recognized. The importance of the spleen in the body's immune response to various infections and the potential consequences of its removal have become increasingly recognized over the past 40-50 years [1]. Asplenic individuals can have major difficulties in coping with specific infections where the spleen would normally play a prominent role in protection. The spleen, in particular, is able to filter encapsulated bacteria such as Streptococcus pneumoniae once blood stream invasion has occurred [2]. Its absence results in an increased risk of serious sepsis carrying considerable mortality. The overall incidence of septicaemia remains low, but death rates from overwhelming postsplenectomy infection (OPSI) have been reported to be up to 600 times greater than in the general population, with an estimated lifetime risk for OPSI syndrome of approximately 5\% [3]. Herein we reported three cases of OPSI syndrome and wanted to renew the knowledge about this syndrome.

\section{Case Reports}

Case 1

A 21 year-old-male, who had splenectomy for idiopathic thrombocytopenic purpura 8 years ago, was admitted to our intensive care unit due to fulminant sepsis. On admission, petechiae and ecchymoses characterized the clinical presentation of the patient. Laboratory tests revealed the presence of renal insufficiency and thrombocytopenia with disseminated intravascular coagulation. S. pneumoniae were yielded in blood cultures. The patient died as a result of refractory septic shock. In his medical history, he had been vaccinated with the polyvalent pneumococcal vaccine early

Received on 20 June 2008; revised 13 November 2008.

Address for correspondence: Dr. Gulbin Aygencel. Gazi University Faculty of Medicine, Department of Internal Medicine, Division of Critical Care Medicine, Besevler, 06510, Ankara, Turkey. Phone: +90 312202 5041; +90 312285 1997. E-mail: aygencel@hotmail.com.

The Brazilian Journal of Infectious Diseases

2008;12(6):549-551. (C) 2008 by The Brazilian Journal of Infectious Diseases and Contexto Publishing. All rights reserved. after splenectomy, was on antibiotic prophylaxis for two years after splenectomy, and hospitalized for pneumonia a month before he died.

\section{Case 2}

A 43 year-old-female, who had a splenectomy due to idiopathic thrombocytopenic purpura 15 years previously, was admitted to our intensive care unit with high fever, headache and loss of consciousness. Meningeal irritation findings were positive in her physical examination. Examination of cerebrospinal fluid (CSF) showed pleocytosis, elevated protein and reduction of glucose levels. S. pneumoniae was detected in the CSF and blood cultures. We diagnosed her as having pneumococcal meningitis and OPSI syndrome. This isolate could not be serotyped and no resistance was recorded against penicillin, ceftriaxone or meropenem. Because she was admitted to our intensive care unit due to sepsis, intravenous meropenem $2 \mathrm{~g}$ per 8 hours was started. Unfortunately, she died within 18 hours after admission as a result of septic shock syndrome. She had never been vaccinated with pneumococcal vaccine.

\section{Case 3}

A 47 year-old-female,who had a splenectomy following abdominal trauma 8 years ago, was admitted to our hospital with high fever, headache, and loss of consciousness. Neck stiffness was the prominent finding in her physical examination. Examination of cerebrospinal fluid showed pleocytosis up to $2400 \mathrm{cell} / \mathrm{mm}^{3}$ with $93 \%$ of polymorphonuclear cells and elevated protein to $1,000 \mathrm{mg} / \mathrm{dL}$ and reduction of glucose to $20 \mathrm{mg} / \mathrm{dL}$. S. pneumoniae was isolated from CSF. Serotyping was not performed on this isolate and penicillin resistance was not found. Pneumococcal meningitis was diagnosed and started to treat with intravenous ceftriaxone $2 \mathrm{~g}$ per 12 hours. Because the patient still had high fever at the 7th day of therapy, Cranial Magnetic Resonance Imaging was performed. Mastoiditis and early brain abscess were found. Ceftriaxone therapy was switched to meropenem therapy. At the end of the therapy, she made a full recovery 
Figure 1. Gram-positive diplococcus in CSF (from Case 3).

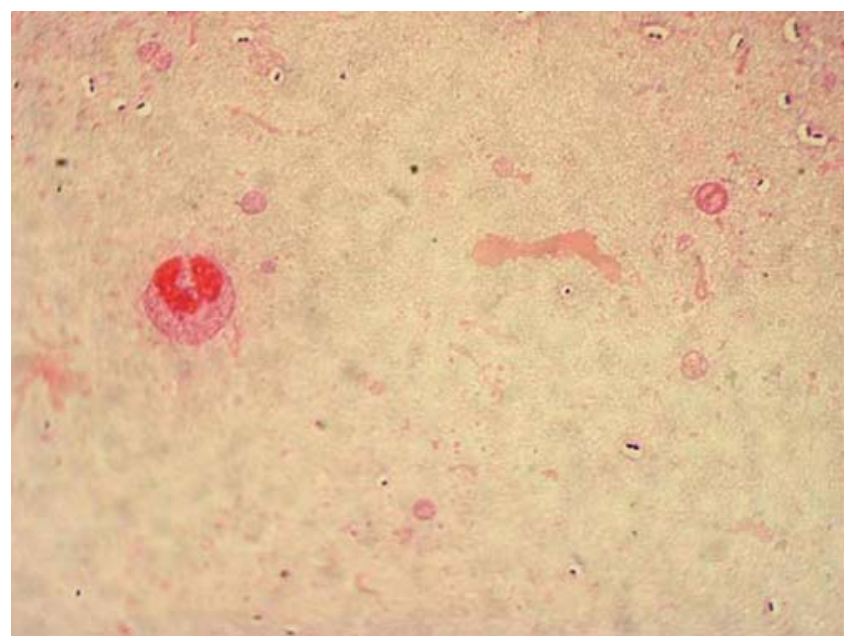

without sequelae. In this case, also there was no vaccination or antibiotic prophylaxis history after splenectomy.

\section{Discussion}

The spleen is crucial to the host response to infection by clearing polysaccharide-encapsulated bacteria. This response involves the clearing of pathogens from the bloodstream as well as the rapid production of specific antigens. Splenectomy results in an increased risk of septic complications associated with a high mortality rate, the most serious being the development of OPSI. Although meningitis or pneumonia will accompany OPSI in $50 \%$ of cases, in many patients there is no obvious site of bacterial colonization and a cryptic source originating in the nasopharynx is postulated. The prodromal stage may be mild and nonspecific with flu-like symptoms [4]. These symptoms may include fever, malaise, myalgias, headache, vomiting, diarrhea, and abdominal pain. This initial prodrome may be followed by rapid evolution to full blown bacteremic septic shock accompanied by hypotension, anuria, and clinical evidence of disseminated intravascular coagulation, thus making this syndrome a true medical emergency. The mortality rate is 50 $70 \%$ despite aggressive therapy that includes intravenous fluids, antibiotics, vasopressors, steroids, heparin, packed red blood cells, platelets, cryoprecipitates, and fresh frozen plasma $[5,6]$.

Splenectomized patients are at risk of life-threatening sepsis. Major factors for stratifying risk include the age at which splenectomy occurs, the subsequent time interval from splenectomy, the reason for splenectomy, and the overall immune status of the patient.

Splenectomy performed for a hematologic disorder, such as thalassemia or lymphoma appears to carry a higher risk than splenectomy performed as a result of trauma [7]. The time interval from the date of splenectomy is also an important risk factor. Several studies have shown that $50 \%$ to $70 \%$ of admissions to a hospital for serious infections occur within
Figure 2. Mastoiditis on the left side (Cranial MRI findings of Case 3).

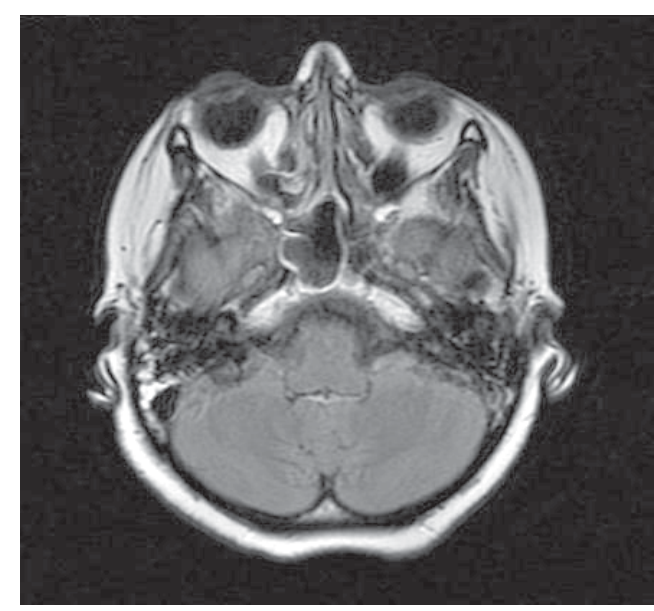

the first 2 years following splenectomy. Thirty-three percent of postsplenectomy pneumococcal infections and $42 \%$ of OPSI occurred $>5$ years postsplenectomy. There are also individual case reports of OPSI occurring $>40$ years after splenectomy [8].

Sepsis in asplenic patients can occur with any organism, be it bacteria, virus, fungus, or protozoan, however encapsulated organism are frequently associated with sepsis in splenectomized patients. Most instances of serious infection are due to encapsulated bacteria such as pneumococcus. Pneumococcal infections have actually accounted for $50 \%$ to $90 \%$ of cases reported in the literature and may be associated with a mortality of up to $60 \%$. Haemophilus influenzae type B, Neisseria meningitidis, and group A streptococci have accounted for an additional 25\% infections [9].

Despite appropriate antibiotics and intensive therapeutic intervention, the overall mortality in older published studies for established cases of OPSI varied from $50 \%$ to $70 \%$. More recent information suggests that when informed patients seek medical attention promptly, the mortality may be reduced to the range of $10 \%$. Of those patients who die, $>50 \%$ die within the first $48 \mathrm{hrs}$ of hospital admission. In those patients who survive, other sequelae, in addition to gangrene and amputation, have included deafness associated with either meningitis or mastoid osteomyelitis, and aortic insufficiency secondary to endocarditis [10].

Treatment of OPSI is generally agressive due to the serious nature of the condition and associated mortality. Therefore prevention of OPSI is extremely important for immune compromised patients who have undergone splenectomy. Preventive strategies fall into three major categories; education, immunoprophylaxis and chemoprophylaxis [11].

Patient education represents a mandatory strategy in attempting to prevent OPSI. Studies have shown that from $11 \%$ to $50 \%$ of postsplenectomy patients remain unaware of their increased risk of serious infection or the appropriate health precautions that should be taken. Patients should 
understand the potential seriousness of OPSI and the rapid possible time course of progression [12].

Functionally or anatomically asplenic patients are at increased risk of infection from encapsulated organism compared to the general population. The most dangerous pathogen for asplenic individuals is unquestionably $S$. pneumoniae. Vaccines available for the most common organisms include the 23 valent pneumococcal polysaccharide vaccine, 7 valent protein conjugated pneumococcal vaccine, the $H$. influenzae type B vaccine, and the meningococcal vaccine. The polysaccharide-based pneumococcal vaccine is recommended for all adults at increased risk of pneumococcal infection, and particularly the asplenic patients. A least $87 \%$ of episodes were caused by this organism, yet only $31 \%$ of patients had received pneumococcal vaccine before OPSI. This is important when considering that $75 \%$ of infecting strains belonged to serotypes present in the current 23 valent polysaccharide vaccine. Immunization has become accepted as an integral part of the preventive strategy against serious infection in the asplenic population, but sporadic cases of pneumococcal and other vaccine failures have been reported in appropriately immunized persons. In addition, bacteria other than pneumococcus account for at least $50 \%$ of cases of OPSI. For these reasons, vaccination by itself should never be allowed to confer a false sense of security [13].

Most authorities recommend antibiotic prophylaxis for asplenic or hyposplenic children, especially for the first 2 yrs after splenectomy. Some investigators advocate continuing chemoprophylaxis in children for at least 5 years, or until the age of 21. The value of such an approach in older children or in adults has never been adequately evaluated in a clinical trial. Compliance is a problem with long-term prophylaxis in adults [14].

Based on epidemiological reports, penicillin-resistant strains are becoming more prevalent throughout Turkey. At the end of 2004, approximately $40 \%$ of strains were resistant to penicillin and nearly one-fifth of all resistant isolates presented high-level penicillin resistance. Although the incidence of $S$. pneumoniae strains resistant to other antimicrobial agents is increasing, ceftriaxone and cefotaxime resistance levels are still low. Consequently, the treatment of choice in S. pneumoniae invasive disease is third-generation cephalosporins due to the low prevalence of resistance. Also according to results of these epidemiological reports, serotyping of penicillin intermediate and resistant $S$. pneumoniae isolates revealed serotypes 1 , $3,6,8,9,14,15,19,20$, and 23 . The serotypes detected among the penicillin intermediate and resistant pneumococci in our country, are among the serotypes covered by 23 -valent and 7 -valent conjugated pneumococcal vaccine, indicating the possible preventive role of the vaccine against pneumococcal infections [15,16].

In our cases, $S$. pneumoniae was responsible for these three infections. One of the three cases could be considered possible vaccine failure, whereas in the other two cases were not present vaccination histories. In case 1 , possible vaccine failure was also doubtful, because either the causative strain belonged to serotype not known in the patient or the immunization had been given more than 5 years. But it was certain that medical staff had not administered efficient preventive strategies for these three cases after splenectomy. Case 3 was also a good example for complication of OPSI syndrome with a brain abscess.

In conlusion, splenectomized patients have been recognized as immunocompromised hosts, and carry high morbidity and mortality risk from fulminant bacterial infections. Therefore, emergency treatment is important to reduce high mortality in such infections.

The aggressive approach to splenic preservation is a way of reducing the risk of subsequent overwhelming postsplenectomy sepsis, but in the case of necessary splenectomy, the patient should be given long-term prophylactic antibiotics and polyvalent pneumococcal vaccine.

\section{References}

1. Shaw J.H.F., Print C.G. Postsplenectomy sepsis. Br J Surg 1989;76:1074-81.

2. Jenks P.J., Jones E. Infections in asplenic patients. Clin Microbiol Infect 1996;1:266-72.

3. Lynch A.M., Kapila R. Overwhelming postsplenectomy infection. Infect Dis Clin North Am 1996;10:693-707.

4. Brigden M.L., Pattullo A.L. Prevention and management of overwhelming postsplenectomy infection-an update. Crit Care Med 1999;27:836-42.

5. Chaikof E.L., McCobe C.J. Fatal overwhelming postsplenectomy infection. Am J Surg 1985;149:534-9.

6. Brigden ML. Overwhelming postsplenectomy infection still a problem. West J Med 1992;157:440-3.

7. Weintraub L.R. Splenectomy: Who, when and why? Hosp Pract 1994;29:27-34

8. Cullingford G.L., Watkins D.N., Watts A.D., et al. Severe late postsplenectomy infection. Br J Surg 1991;78:716-21.

9. Anonymous: Guidelines for the prevention and treatment of infection in patients with an absent or dysfunctional spleen. Working part of the British Committee for standarts in Haematology. BMJ 1996;312:430-4.

10. Styrt B. Infection associated with asplenia. Risks, mechanisms, and prevention. Am J Med 1990;88:33N-42N.

11. Williams D.N., Kaur B. Postsplenectomy care. Strategies to decrease the risk of infection. Postgrad Med J 1996;100:195-205.

12. White K.S., Covington D., Churchill P., et al. Patient awareness of health precautions after splenectomy. Am J Infect Control 1991;19:36-41.

13. Kind E.A., Craft C., Fowles J.B., et al. Pneumococcal vaccine administration associated with splenectomy missed opportunities. Am J Infect Control 1998;26:418-22.

14. Finch R.G., Read R. Lifelong penicillin may be ineffective. BMJ 1994;308:132

15. Yalcýn I., Gurler N., Alhan E., et al. Serotype distribution and antibiotic susceptibility of invasive Streptococcus pneumoniae disease isolates from children in Turkey, 2001-2004. Eur J Pediatr 2006;165:654-7.

16. Gur D., Ozalp M., Sumerkan B., et al. Prevalence of antimicrobial resistance in Haemophilus influenzae, Streptococcus pneumoniae, Moraxella catarrhalis and Streptococcus pyogenes; results of a multicentre study in Turkey. Intern J Antimicrob Agents 2002;19:207-11. 